\title{
Location of Cerebral Microbleeds May Predict Subsequent Stroke after Transient Ischemic Attack
}

\author{
Jae-Sung Lim, ${ }^{a}$ Hyung-Min Kwon, ${ }^{b}$ Young-Seok Lee ${ }^{\mathrm{b}}$ \\ aDepartment of Neurology, Hallym University Sacred Heart Hospital, Anyang, Korea \\ bDepartment of Neurology, Seoul National University Boramae Hospital, Seoul, Korea
}

\section{Dear Sir:}

The prognostic significance of cerebral microbleeds (CMBs) in ischemic stroke has previously been investigated. ${ }^{1,2}$ However, there are as yet no reports regarding the detailed relationship between the CMB location and recurrent stroke following a transient ischemic attack (TIA). We thus aimed to investigate the association between CMB location and subsequent stroke following a TIA in detail.

We conducted a hospital-based, multi-center, prospective cohort TIA study (Korean TIA eXpression study, KTX). ${ }^{2}$ Consecutive patients with TIA were enrolled from 11 university hospitals from July 1, 2010 through December 31, 2012. TIA was defined using classic time-based criteria as a focal neurologic deficit lasting less than 24 hours. Patients 40 years or older who were admitted within 24 hours of symptom onset and underwent magnetic resonance imaging, including diffusion-weighted imaging and magnetic resonance angiography, were included in the study. This study was approved by the institutional review boards of all participating hospitals. Written informed consent was obtained from all patients or from their legally authorized representatives.

The locations of the CMBs were classified according to the Microbleed Anatomical Rating Scale. ${ }^{3}$ We further trichotomized the CMBs as strictly lobar, strictly deep, and mixed based on their locations. Along with CMB location, other neuroimaging variables, including acute ischemic lesions in diffusion-weighted images (single or multiple), prior territorial infarction, lacunar infarction, intracerebral hemorrhage, and white matter hyperintensities were also investigated. Crescendo TIA was defined as at least 3 similar attacks occurring within 7 days from the index
TIA. ${ }^{4}$ Risk stratification scores for $A B C D^{2}$ and $A B C D^{3}-I$ scores were also determined. Detailed descriptions of the clinical and neuroimaging variables were presented in a previous study. ${ }^{2}$ The prognostic significance of CMB location was investigated using the primary endpoint of subsequent stroke within 90 days of index TIA occurrence. Subsequent stroke was assessed at an outpatient clinic or by telephone interview using a structured questionnaire. If necessary, we reviewed medical records to confirm the diagnosis of subsequent stroke. Lesion-positive TIA was not included as an outcome event.

We analyzed the data using analysis of variances for continuous variables and the $\chi^{2}$ test and Fisher's exact test for categorical variables. In multivariable analyses, we estimated hazard ratios for subsequent stroke after TIA and calculated 95\% confidential intervals (Cls) with adjustments for possible confounders using Cox proportional hazards models. We reduced the number of covariates using the composite risk score $A B C D^{3}-$ I rather than using individual clinical and neuroimaging variables. ${ }^{2}$ Statistical analyses were performed with SPSS statistical software version 21 (IBM SPSS Inc.). A two-sided $P<0.05$ was considered as the minimum level for statistical significance.

Of the 521 consecutive patients with TIA that were screened during the study period, 500 (96.0\%) were enrolled. Twenty-one patients were excluded due to protocol violation or consent withdrawal. ${ }^{2}$ Recurrent ischemic stroke within 90 days of the TIA occurred in 25 patients. The baseline characteristics of the study subjects are presented in Supplemental Table 1. Strictly lobar CMBs were observed in $16.3 \%$, strictly deep CMBs were found in $53.5 \%$, and mixed CMBs were seen in $30.2 \%$ of the patients. The frequently affected sites were the basal ganglia followed by the 
Table 1. Multivariable Cox proportional hazards models for CMB location and subsequent stroke after TIA

\begin{tabular}{|c|c|c|}
\hline & Hazard ratio $(95 \% \mathrm{Cl})$ & $P$ value \\
\hline \multicolumn{3}{|l|}{ Model 1} \\
\hline$A B C D^{3}$-I score $8-13^{*}$ & $1.42(0.57-3.56)$ & 0.45 \\
\hline Crescendo TIA & $2.11(0.64-6.98)$ & 0.22 \\
\hline Lacunes & $1.46(0.57-3.79)$ & 0.43 \\
\hline White matter hyperintensities & $1.53(0.60-3.88)$ & 0.37 \\
\hline $\mathrm{CMBs}$, total number & $0.92(0.80-1.06)$ & 0.26 \\
\hline $\mathrm{CMBs}$, strictly lobar ${ }^{+}$ & $3.82(0.41-35.58)$ & 0.24 \\
\hline CMBs, strictly deep ${ }^{\dagger}$ & $1.23(0.16-9.58)$ & 0.84 \\
\hline $\mathrm{CMBs}_{\text {, mixed }}{ }^{+}$ & $16.41(3.44-78.26)$ & $<0.01$ \\
\hline \multicolumn{3}{|l|}{ Model 2} \\
\hline$A B C D^{3}-1$ score $8-13^{*}$ & $1.62(0.68-3.89)$ & 0.28 \\
\hline Lacunes & $1.42(0.55-3.68)$ & 0.47 \\
\hline White matter hyperintensities & $1.56(0.62-3.89)$ & 0.34 \\
\hline CMBs, total number & $0.92(0.78-1.07)$ & 0.27 \\
\hline $\mathrm{CMBs}$, strictly lobar ${ }^{+}$ & $5.54(0.63-48.90)$ & 0.12 \\
\hline $\mathrm{CMBs}$, strictly deep ${ }^{+}$ & $1.24(0.16-9.76)$ & 0.84 \\
\hline $\mathrm{CMBs}_{\text {, mixed }}{ }^{+}$ & $19.90(4.04-98.07)$ & $<0.01$ \\
\hline \multicolumn{3}{|l|}{ Model 3} \\
\hline$A B C D^{3}-1$ score $8-13^{*}$ & $1.63(0.68-3.92)$ & 0.27 \\
\hline White matter hyperintensities & $1.70(0.70-4.10)$ & 0.24 \\
\hline CMBs, total number & $0.92(0.80-1.07)$ & 0.30 \\
\hline $\mathrm{CMBs}$, strictly lobar ${ }^{+}$ & $6.06(0.70-52.80)$ & 0.10 \\
\hline $\mathrm{CMBs}$, strictly deep ${ }^{+}$ & $1.26(0.16-9.92)$ & 0.83 \\
\hline $\mathrm{CMBs}_{\text {, }}$ mixed $^{+}$ & $19.83(4.21-93.46)$ & $<0.01$ \\
\hline
\end{tabular}

TIA, transient ischemic attack; CMBs, cerebral microbleeds.

${ }^{*}$ Compared to the lowest category (scores $\left.0-7\right) ;{ }^{+}$Compared to patients without $\mathrm{CMBs}$.

thalamus and temporo-occipital lobar areas (Supplemental Table 2). Recurrent stroke occurred in $4.2 \%$ of patients without CMBs, $14.3 \%$ of patients with strictly lobar CMBs, $4.3 \%$ of those with strictly deep $\mathrm{CMBs}$, and $38.5 \%$ of those with mixed CMBs.

The unadjusted hazard ratio of mixed CMB locations for subsequent stroke following TIA was $12.94(95 \% \mathrm{Cl}$ 4.77-35.13, $P$ value $<0.01$ ). According to our multivariable analysis results, mixed CMBs were significantly associated with a risk of recurrent stroke after TIA independently of the number of CMBs (adjusted hazard ratio $19.83,95 \% \mathrm{Cl} 4.21-93.46$ ). We tested several multivariable models and still observed a significant effect of mixed CMB locations on subsequent stroke (Table 1).

Our study reveals that CMBs with mixed locations (lobar and deep) are associated with subsequent stroke following TIA independently of the number of CMBs. The exact mechanism underpinning this association is still unknown. We suggest that the presence of mixed CMB locations may reflect the existence of vulnerable cerebral milieus rather than risk itself.

Cortical microinfarcts at autopsy are associated with CMBs in patients with cerebral amyloid angiopathy, which may reflect possible pathophysiological links between CMBs and cerebral ischemia. ${ }^{5}$ Chronic hypertension may disrupt cerebral autoregu- lation of the superficial perforating arteries of pial origin, which results in the exposure of these small arteries to excessive pressure and subsequent damage to smooth muscle cells. ${ }^{6}$ Disruption of the internal lamina and thin adventitia of the lobar or corticosubcortical portion of pial arterioles is associated with white matter pathology in cerebral autosomal dominant arteriopathy with subcortical infarcts and leukoencephalopathy. ${ }^{7}$ A recent intriguing study regarding the pathogenesis of $\mathrm{CMBs}$ revealed that lobar CMBs were associated with both amyloid-related pathologies and subcortical small vessel disease, whereas deep-seated CMBs were only associated with subcortical small vessel disease. ${ }^{8}$ Based on these findings, mixed CMB locations may reflect widespread pathological changes of the cerebral vasculature induced by chronic vascular damage and a hostile milieu associated with subsequent ischemic events.

Our study had the limitation of having a small number of main outcome events, which may lead our multivariable models to be over-fitted. Our findings should thus be validated in further large-scale clinical studies. Nevertheless, our study's strength is that it investigated the association between CMB location and subsequent stroke in a multicenter, prospective cohort with TIA.

In conclusion, mixed CMB location was associated with subsequent stroke after TIA occurrence, while strictly lobar or deep CMBs were not. Our data on CMB location imply that the local milieu reflected by the CMBs may predict future ischemic stroke occurrence in patients with TIAs. The exact mechanism underlying these associations should be further investigated.

\section{References}

1. Charidimou A, Kakar P, Fox Z, Werring DJ. Cerebral microbleeds and recurrent stroke risk: systematic review and meta-analysis of prospective ischemic stroke and transient ischemic attack cohorts. Stroke 2013;44:995-1001.

2. Lim JS, Hong KS, Kim GM, Bang OY, Bae HJ, Kwon HM, et al. Cerebral microbleeds and early recurrent stroke after transient ischemic attack: results from the Korean Transient Ischemic Attack Expression Registry. JAMA Neurol 2015;72:301-308.

3. Gregoire SM, Chaudhary UJ, Brown MM, Yousry TA, Kallis C, Jager HR, et al. The Microbleed Anatomical Rating Scale (MARS): reliability of a tool to map brain microbleeds. Neurology 2009;73:1759-1766.

4. Rothrock JF, Lyden PD, Yee J, Wiederholt WC. 'Crescendo' transient ischemic attacks: clinical and angiographic correlations. Neurology 1988;38:198-201.

5. Lauer A, van Veluw SJ, William CM, Charidimou A, Roongpiboonsopit $D$, Vashkevich $A$, et al. Cerebral microbleeds on clinical MRI are associated with cortical microinfarcts on au- 
topsy in cerebral amyloid angiopathy. Proceedings of the VASCOG World 2015, P22-6.

6. Werring DJ. Cerebral microbleeds: pathophysiology to clinical practice. Cambridge, UK: Cambridge University Press, 2011.

7. Miao $\mathrm{Q}$, Paloneva T, Tuominen S, Poyhonen M, Tuisku S, Viitanen $M$, et al. Fibrosis and stenosis of the long penetrating cerebral arteries: the cause of the white matter pathology in cerebral autosomal dominant arteriopathy with subcortical infarcts and leukoencephalopathy. Brain Pathol 2004;14:358364.

8. Park JH, Seo SW, Kim C, Kim GH, Noh HJ, Kim ST, et al. Pathogenesis of cerebral microbleeds: in vivo imaging of amyloid and subcortical ischemic small vessel disease in 226 individuals with cognitive impairment. Ann Neurol 2013;73:584-593.
Correspondence: Yong-Seok Lee

Department of Neurology, Seoul National University Boramae Hospital, 20 Boramae-ro 5-gil, Dongjak-gu, Seoul 07061, Korea

Tel: +82-2-870-2472, Fax: +82-2-870-2709

E-mail: mercades@snu.ac.kr

Received: April 27, 2016

Revised: April 27, 2016

Accepted: April 28, 2016

This study was supported by a grant from the SNU R\&DB Foundation, Republic of Korea (RES10-RF136, 800-20100046, 800-20100055).

The authors have no financial conflicts of interest. 